\title{
56. Kongress der Deutschen Gesellschaft für Pneumologie und Beatmungsmedizin e.V.
}

\author{
Anlässlich des diesjährigen DGP-Kongresses, der vom \\ 18. bis 21. März 2015 im CityCube in Berlin stattfand, geben \\ die 15 wissenschaftlichen Sektionen in dieser und der nächsten \\ Ausgabe der „Pneumologie“ einen aktuellen Überblick über \\ zurückliegende Aktivitäten, personelle und inhaltliche \\ Neuerungen sowie einen Ausblick auf kommende Termine \\ und Schwerpunkte in den jeweiligen Fachbereichen.
}

\section{Allergologie und Immunologie}

Die Sektion „Allergologie und Immunologie“ ist zurzeit mit 534 Mitgliedern die fünftgrößte Sektion in der DGP. Die Lunge ist ein zentrales Organ im großen Spektrum allergologischer und immunologischer Erkrankungen. Die Entwicklung von Allergien spielt gerade in Industrieländern eine immer bedeutendere Rolle und dabei ist Asthma bronchiale das am häufigsten auftretende Krankheitsbild im Kindesalter. In den letzten Jahren wurde zunehmend deutlich, dass bestimmte herausragende Asthma-Merkmale die Einteilung in Asthma-Phänotypen ermöglicht und diese - mittlerweile durch eine gute klinische Studienlage belegt - individuell mittels sog. Biologika als Add-on-Therapie zur herkömmlichen Standard-Therapie effektiv behandelt werden können. Dies ist gerade im Hinblick auf die schweren therapierefraktären Asthma-Formen, wie z.B. das schwere eosinophile Asthma, von großer Bedeutung.

Kurz nach dem DGP-Kongress in Bremen 2014 begann die Sektion mit den Vorbereitungen für den Kongress 2015. Durch insgesamt 19 Veranstaltungen konnte in Berlin ein weitreichendes Themenspektrum des Schwerpunkts Allergologie und Immunologie präsentiert werden. Neben 2 Postgraduiertenkursen fanden 2 Frühseminare, 13 Symposien, einige freie Vorträge und eine Posterbegehung statt.
Die aktuelle Auswertung der Teilnehmerzahlen in den angebotenen Veranstaltungen verdeutlicht, dass gerade die Themenschwerpunkte „Der Kampf um den eosinophilen Asthmatiker - Wer gewinnt?“, „Zehn Highlights aus der aktuellen klinischen Allergieforschung“, „Hot Topic: obstruktive Lungenerkrankungen“, „Anaphylaxie: Wo stehen wir heute?“, „Asthma bronchiale - Fakten und Visionen“ und „Schimmelpilz-Allergie - Was muß der Pneumologe wissen?“, „Allergologische und immunologische Perlen für Experten“ sowie „Allergieprävention“ mit bis zu 300 Teilnehmern eine große Resonanz fanden und somit den Informationsbedarf der Kongressteilnehmer erfüllten.
Die Förderung und Unterstützung von wissenschaftlich tätigem Nachwuchs ist ein wesentlicher Meilenstein unserer Sektionsarbeit. Junge Wissenschaftler finden in unserer Sektion eine Heimat, die ihnen erlaubt, ihre Forschungsergebnisse erstmals vorzustellen und mit erfahrenen Kollegen zu diskutieren. Die Einreichung von wissenschaftlichen Abstracts in unserer Sektion für den DGP-Kongress 2015 in Berlin zeigt, dass sich auf dem Gebiet der Allergologie und Immunologie, insbesondere auf dem Gebiet der TH1-Zellen aber auch auf dem Gebiet der Einflussnahme von viralen und bakteriellen Infektionen auf die entsprechende Immunantwort, z.B. mittels TLR (Toll-like-Receptors) interessante, neue wissenschaftliche Zusammenhänge auftun, die zu mehr Verständnis beitragen und in Zukunft neue klinische Relevanz haben könnten. Da liegt es nahe, eine gesonderte Herbsttagung für Grundlagen- und klinische Forschung zu etablieren. Wir freuen uns deshalb sehr, dass die Sektion 14 (Zellbiologie) ihre Tore für unsere wissenschaftlichen Sektionsmitglieder öffnet und wir von nun an eine gemeinsame Herbsttagung anbieten können. Unsere wissenschaftlich tätigen Sektionsmitglieder werden hierbei unter der Leitung von PD Dr. Jürgen Knobloch, Bochum, und Prof. Marco Idzko, Freiburg, durch die zukünftig gemeinsame Herbsttagung begleitet. Beide Sektionen haben diesem wissenschaftlichen Bündnis zugestimmt. Die Sektion Zellbiologie bleibt hierbei die leitende Sektion dieser Tagung. Alle, insbesondere junge Wissenschaftler auf dem Gebiet Aller-

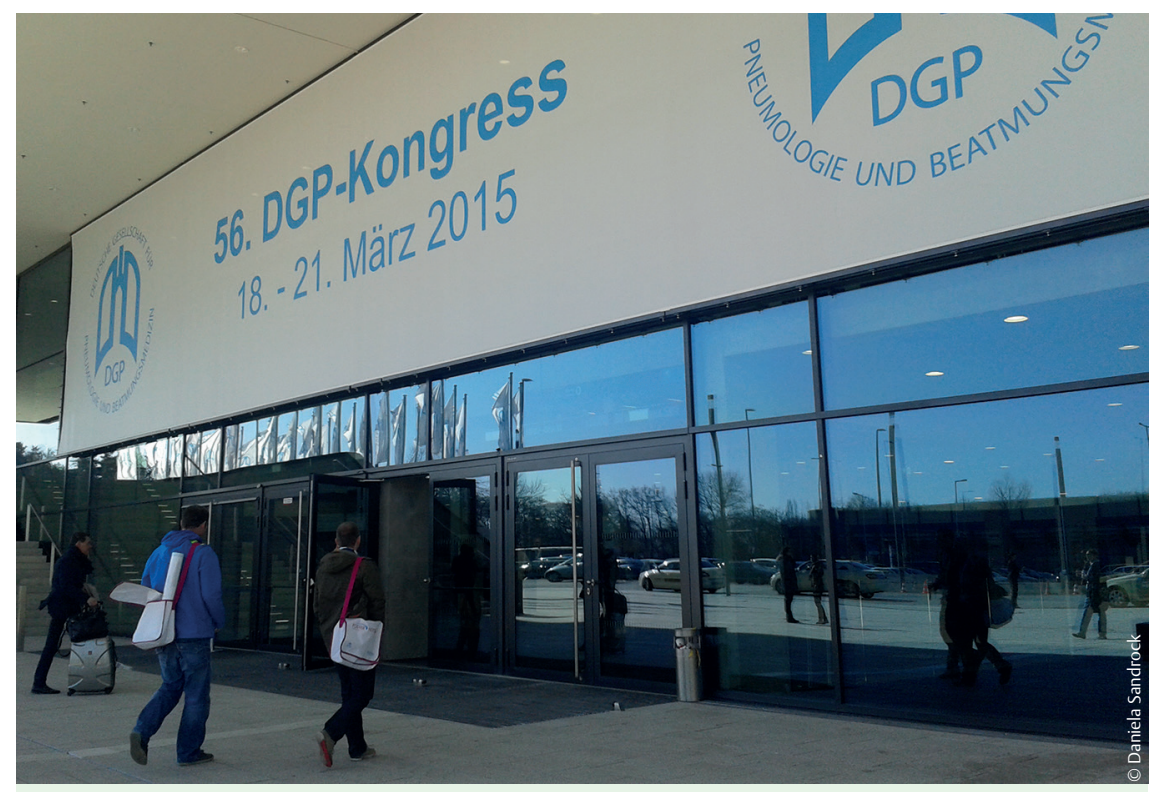

Auf dem diesjährigen DGP-Kongress gaben rund 591 Referenten ihr Wissen im Rahmen von fast 200 Veranstaltungen (Seminare, Kurse, Symposien und Vorträge) weiter. 
gologie und Immunologie, sind herzlich eingeladen, ihre Forschungsergebnisse auf dem kommenden gemeinsamen Herbsttreffen zu präsentieren.

Ziel unserer Sektion ist es, allergologische und immunologische Erkenntnisse in den Zusammenhang des großen Spektrums von Lungenerkrankungen zu stellen und dies auf wissenschaftlich fundiertem Boden einem breiten Kreis der in Universitäten, Krankenhäusern oder im niedergelassenen Bereich arbeitenden Kollegen an die Hand zu geben. Dafür sei jedem einzelnen aktiv tätigen Sektionsmitglied, das dieses Ziel durch seine Mitarbeit im Auge hat, auch an dieser Stelle herzlich gedankt. Wünschenswert bleibt eine aktive Mitarbeit unsere Sektion bei der Ausrichtung zukünftiger Fortbildungen durch die DGP, wie bei der Winterakademie durch die Besetzung von Themen (z.B. der Immuntherapie).

Beim Treffen des gemeinsamen Bundesausschusses in Berlin im Januar dieses Jahres wurde die DGP durch die Sektionssprecherin der Sektion 1, Prof. Andrea Koch, Bochum, vertreten. Ziel dieses Treffens war eine Expertendiskussion mit den einzelnen Fachvertretern zur Planung von 2 Studien: zum einen zum Stellenwert von NO im Vergleich zur unspezifischen bronchialen Provokation bei der Diagnostik von Asthma sowie zum anderen zu Schwangerschaft und Asthma.

Die DGP stellt alle 3 Jahre einen German Delegate der European Respiratory Society, der die Belange der DGP bei der ERS vertritt. Derzeit obliegt dieses Amt Prof. Andrea Koch, Bochum. Sie werden demzufolge zukünftig ein „ERS-Info-Fenster“ auf der Website der DGP vorfinden. Dort können zukünftig alle aktuellen ERS-Informationen eingesehen werden. Zwei hochaktuelle Informationen hierzu schon vorweg:

- Sie haben erstmals die Möglichkeit eine doppelte Mitgliedschaft von DGP und ERS durch einen nur geringfügig zusätzlichen Beitrag von $20 €$ zum DGPBeitrag zu erwerben.

- Prof. Oliver Eickelberg, München, wird sich zur Wahl des Science Council Chair der ERS aufstellen lassen. Er benötigt unsere vielzählige Unterstützung, um zukünftig unsere landesweiten wissenschaftlichen Interessen innerhalb der ERS zu stärken.

Darüber hinaus konnte die Sektion das Organ unserer Gesellschaft, die Zeitschrift
„Pneumologie“, mit verschiedenen Beiträgen in ihrer Arbeit unterstützen. Es wurden u. a. 2014 eine Fort- und Weiterbildungsreihe „Pneumologische Erkrankungen und gastroösophagealer Reflux" von P. Kardos sowie ein Leserbrief von N. K. Mülleneisen zum Thema „Wirrwarr bei Innovationsdevices gefährdet Patientensicherheit" veröffentlicht. Darüberhinaus erschienen Originalarbeiten aus unserer Sektion zu „Versorgung von Asthmapatienten mit Kombinationstherapie aus ICS und LABA - eine GKV-Daten-Analyse“ von H. Friedel und J. C. Virchow, „Assessment of the Effect of Pharmacotherapy in Common Cold / Acute Bronchitis - the Bronchitis Severity Scale (BSS)“ und ein Beitrag zu „GINA 2014, Licht und Schatten“, beide Beiträge federführend von P. Kardos. Zudem erschien ein Artikel „Disease-Modifikation und Dauer einer Omalizumab-Therapie bei Patienten mit schwerem allergischen Asthma“ von J. Schreiber et al.

Ergänzt wurden die Beiträge 2014 von M. Lommartzsch et al. zum Thema „Severe asthma: Definition, Diagnosis and Treatment" im Deutschen Ärzteblatt, sowie durch die „S3-Leitlinie Allergieprävention - Update 2014“ (AWMF online), die durch die DGP, vertreten von unserem Sektionsmitglied Dr. Horst Müsken, Bad Lippspringe, in Zusammenarbeit mit der Deutschen gesellschaft für Allergologie und klinische Immunologie und der Deutschen Gesellschaft für Kinder- und Jugendmedizin u.v.m. mitgestaltet wurde.

Die Bedeutung des Schwerpunkts Allergologie und Immunologie in der Pneumologie stößt innerhalb der politischen Entscheidungsträgern sowie des wissenschaftlichen Nachwuchses und ebenso bei der gesamten Ärzteschaft und der pneumologisch-assoziierten Heilberufe auf großes Interesse. Die Sektion weiß um ihre Verantwortung gegenüber ihrem wissenschaftlichen Nachwuchs, aber auch gegenüber der Vermittlung von klinischen Positionen, die direkte Auswirkungen auf die praktische allergologische Tätigkeit von Pneumologen und ärztlichem Assistenzpersonal sowie auch auf deren Ausbildung hat. Eine breite Beteiligung unserer Sektion innerhalb der Arbeit in der DGP ist daher auch in Zukunft von besonderer Bedeutung. Sie sind eingeladen, sich aktiv daran zu beteiligen.

Prof. Andrea Koch, Bochum, und Dr. Ute Lepp, Stade
Sektion 2

\section{Endoskopie}

Das zurückliegende Jahr war aus wissenschaftlicher Sicht ein sehr erfolgreiches Jahr für die Sektion 2. Die deutsche Multicenter-Studie zur transbronchialen Kryobiopsie (Kryo-TBB) bei interstitiellen Lungenerkrankungen (ILD) mit mehr als 300 Patienten konnte abgeschlossen werden. Die Folgestudie zur Bestimmung der Wertigkeit der Kryo-TBB wurde mittlerweile erfolgreich initiiert. Auch im Schwerpunkt der endoskopischen Lungenvolumenreduktion beteiligten sich zahlreiche Zentren der Sektion. So wurde die Patientenrekrutierung einer randomisiert-kontrollierten Studie zur Thermoablation (STEPUP) bei Patienten mit oberlappenbetontem Lungenemphysem ebenfalls Ende 2014 vollendet, eine randomisiert-kontrollierte Studie zur Ventilimplantation bei Patienten mit homogenem Lungenemphysem (IMPACT) begonnen.

Die zahlreichen wissenschaftlichen Aktivitäten zeigten sich auch beim diesjährigen Jahreskongress der DGP. Neben der Durchführung und Beteiligung an zahlreichen Symposien und PostgraduiertenKursen wurden insgesamt 28 Abstracts akzeptiert und in freien Vorträgen und 2 Posterbegehungen in Berlin präsentiert.

Auf der Sektionsitzung des Jahreskongresses wurden 2 neue Sektionsprecher gewählt. Prof. Ralf Eberhardt, Heidelberg, und Dr. Manfred Wagner, Nürnberg, vertreten in den nächsten 2 Jahren die Sektion. Darüber hinaus wurde einstimmig die Einrichtung einer Arbeitsgruppe 2.1 „Klinische Zytologie“ innerhalb der Sektion 2 beschlossen.

Neben den zahlreichen, in dem letzten Jahr veranstalteten, nationalen und internationalen Kursen zur diagnostischen und interventionellen Bronchoskopie sowie zur internistischen Thorakoskopie, beteiligten sich die Mitglieder der Sektion auch wieder aktiv an der Planung und Durchführung der europäischen Jahreskongresse der European Respiratory Society (ERS) und der European Association for Bronchology and Interventional Bronchology (EABIP).

Prof. Ralf Eberhardt, Heidelberg, und Dr. Manfred Wagner, Nürnberg 


\section{Sektion 3}

\section{Arbeitsmedizin, Epidemiologie, Umwelt- und Sozialmedizin}

Die Diagnostik und Prävention arbeitsund umweltbedingter Erkrankungen sowie deren Versorgung, Rehabilitation und Kompensation stehen in den Fächern $\mathrm{Ar}$ beitsmedizin, Sozialmedizin, Umweltmedizin und Epidemiologie im Vordergrund. Eine wesentliche Aktivität der Sektionsmitglieder ist hier die Mitwirkung an Leitlinien und Empfehlungen zu diesen Themen. Im Berufskrankheiten(BK)-Verfahren stellen diese Leitlinien und Empfehlungen die Grundlage für die Diagnostik und Beurteilung von Folgezuständen dar und dienen der Gleichbehandlung der Versicherten. Derzeit wird die im Jahr 2008 erstmals erstellte S2-Leitlinie Quarzstaublungenerkrankung (Silikose), Diagnostik und Begutachtung der BK Nr. 4101 unter Federführung unserer Sektion über- arbeitet. An der Überarbeitung der Leitlinie Spirometrie der Atemwegsliga hat unsere Sektion ebenfalls mitgewirkt. Eine besondere Herausforderung wird nun deren Umsetzung in der Bewertung der Minderung der Erwerbsfähigkeit in der BK-Begutachtung sein. Da uns die Prävention sehr wichtig ist, hat unsere Sektion auch an der 2014 fertiggestellten S3-Leitlinie Tabakentwöhnung bei COPD mitgearbeitet.

Das Programm der Deutschen Gesetzlichen Unfallversicherung (DGUV) „Früherkennung asbestverursachter Erkrankungen“ wurde von mehreren Mitgliedern der Sektion geleitet und mitgestaltet. In 2014 konnte mit der Pilotphase an 2 Standorten begonnen werden. Durch das Angebot einer jährlichen arbeitsmedizinischen Untersuchung und Diagnostik mit einer Low-dose-Volumen HR-Computertomografie des Thorax bei Hochrisikopatienten (berufliche Asbestexposition über 10 Jahre und Rauchgewohnheit von mind. 30 Packungsjahren) soll die Diagnostik von Lungenkarzinomen bereits im Frühstadium gelingen, entsprechend der NSLTStudie aus den USA (NEJM 2011).
Die Planung und Durchführung des wissenschaftlichen Programms der Jahrestagung der DGP 2015 in Berlin standen wiederum im Fokus der Sektion. Eine große Herausforderung stellte diesmal die Terminüberschneidung mit der gleichzeitig in München stattfindenden Jahrestagung der Deutschen Gesellschaft für Arbeitsmedizin und Umweltmedizin (DGAUM) dar. Mit großem Engagement beider Fachgesellschaften und der Referenten ist es unserer Sektion gelungen, ein sehr ansprechendes Programm für Berlin zu erstellen. Es zeigte sich wiederum ein hohes Interesse an den seit Jahren etablierten Postgraduiertenkursen zur „Radiologischen Diagnostik pulmonaler Berufserkrankungen“ und dem interaktiven PG-Kurs „Pitfalls: Meet the Expert" mit besonderen Fallbeispielen in der Begutachtung und Diskussion zwischen Arbeitsmedizinern, Pneumologen und Radiologen. Das Frühseminar über die „Thoraxübersichtsaufnahme - der diagnostische Fall“" war erneut ausgebucht.

Das „Berufskrankheiten-Forum“ hat seine erfolgreiche Tradition fortgesetzt. Die sehr hohe Teilnehmerzahl am Samstag- 


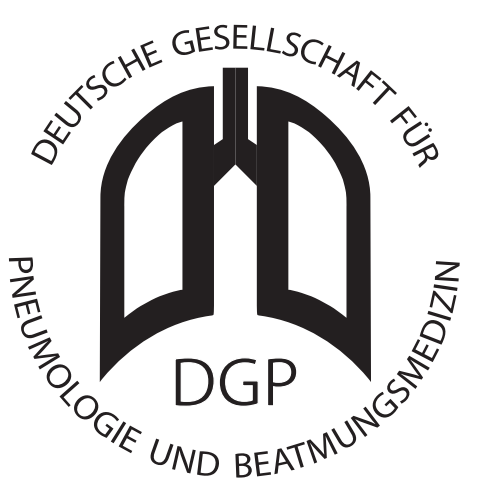

vormittag bestätigte dies eindrucksvoll. Begonnen wurde mit der Übersicht der voraussichtlich wichtigsten Änderungen der Überarbeitung der S2-Leitlinie Quarzstaublungenerkrankung (Silikose), Diagnostik und Begutachtung der BK Nr. 4101, nämlich dem Stellenwert der LD-Volumen-HR-CT und den neuen Lungenfunktionssollwerte. An 2 ausgewählten Kasuistiken konnten Pitfalls in der Begutachtung der BK Silikose dargestellt und diskutiert werden. Weiterhin wurde die Pilotphase des bereits obig beschriebenen Programms der DGUV zur erweiterten Vorsorge von asbestbedingten Erkrankungen vorgestellt. Die Darstellung der Erfahrungen in der radiologischen Qualitätssicherung im Rahmen des erweiterten Vorsorgeangebots rundete das BK-Forum ab.

Im Rahmen des Symposiums „Verkehr und Pneumologie“ in Zusammenarbeit mit der Sektion Schlafmedizin wurde über die Einschätzung und Begutachtung der Fahrtauglichkeit von Personen mit Lungenerkrankungen und Schlafapnoe sowie über schlafbezogene Atmungsstörungen - Einschränkung und Möglichkeiten für privates oder berufliches Reisen - berichtet und diskutiert. Sehr erfreulich war die Sitzung freie Vorträge mit spannenden wissenschaftlichen Vorträgen über verschiedenste Themen bei berufs- und umweltbedingten Lungenerkrankungen. Bei der Postersession wurden sehr gute Poster zu diesen Themen vorgestellt und diskutiert. Im Rahmen der Sektionssitzung haben wir die Themen für den kommenden Kongress in Leipzig besprochen.

Mitglieder der Sektion gestalteten als Erst- und Co-Autoren das Themenheft zu pulmonalen Berufskrankheiten in der Zeitschrift Atemwegs-und Lungenerkrankungen.

Dr. Nicola Kotschy-Lang, Falkenstein, und PD Dr. Astrid Heutelbeck, Göttingen

\section{Infektiologie und Tuberkulose}

Die Sektion blickt auf ein erfolgreiches Jahr zurück. Die Sektion 4 war während des DGP-Kongresses in Berlin mit einem Postgraduiertenkurs, 10 Symposien, weitere Symposien gemeinsam mit dem Deutschen Zentralkomitee zur Bekämpfung der Tuberkulose (DZK), freien Vorträgen und 2 Postersitzungen vertreten.

Das Kompetenznetz CAPNETZ hat nach Überführung in eine Stiftung seine Aktivitäten auf dem klinischen Gebiet der Erforschung der ambulant erworbenen Pneumonie weiter etabliert. Das Netzwerk wurde inzwischen durch einen pädiatrischen Teil ergänzt. Im Berichtsjahr wurden mehrere Studien aus der CAPNETZ-Gruppe in internationalen Journalen publiziert, u.a. zur Diagnostik respiratorischer Virusinfektionen und zur Epidemiologie atypischer Bakterien wie Mykoplasmen und Chlamydien. Über die CAPNETZ-Plattform wird weiterhin für die wichtige ABACOPD-Studie zur Notwendigkeit einer AntibiotikaTherapie bei einer akuten COPD-Exazerbation rekrutiert. Interessenten aus Praxis und Klinik werden gebeten, sich bei Teilnahmewunsch beim CAPNETZ-Vorstand zu melden. Die Überarbeitung der S3-Leitlinie zur ambulant erworbenen Pneumonie unter Federführung von Prof. Santiago Ewig, Bochum, soll in diesem Jahr abgeschlossen werden.

Die zahlreichen Kooperationen der Sektion 4 werden weiterhin lebhaft ausgestaltet und weiterentwickelt. Hierzu gehören die Sektionen Zellbiologie, Intensiv- und Beatmungsmedizin, Pädiatrische Pneumologie und Pathophysiologie sowie Aerosolmedizin der DGP, die AGs Fort- und Weiterbildung und Atmungstherapeuten, die Deutsche Veterinärmedizinische Gesellschaft (DVG), die Paul-Ehrlich-Gesellschaft, die Deutsche Mykologische Gesellschaft und das DZK.

Nach intensiver Vorbereitung wird im zweiten Quartal 2015 das deutsche Bronchiektasen-Register PROGNOSIS, das von den Sektionsmitgliedern Dr. Jessica Rademacher und Dr. Felix Ringshausen aus Hannover sowie Dr. Andrés de Roux aus Berlin koordiniert wird und an dem auch zahlreiche andere Mitglieder der Sektion beteiligt sind, mit der Rekrutierung begin- nen können. Hier besteht eine sehr fruchtbare Zusammenarbeit mit dem europäischen Bronchiektasen-Register EMBARC, das eine Clinical Research Collaboration (CRC) der ERS ist.

Die Tradition einer gemeinsamen wissenschaftlichen Herbsttagung mit der Sektion Zellbiologie haben wir fortgeführt, dieses mal auch unter Beteiligung der Sektion Pathophysiologie und Aerosolmedizin. Die Ausrichtung der Tagung fand durch die Sektion 4 im Audimax der Universität Lübeck statt und wurde mit ca. 70 Teilnehmern sehr gut besucht. Die Organisation wurde von Dr. Kristina Rohmann und Dr. Daniel Drömann aus unserer Sektion übernommen. Die Keynote-Lecture hielt Prof. Gülsah Gabriel, Heinrich-Pette-Institut Hamburg über „Influenza: a continuous challenge for science and health care“. $\mathrm{Zu}$ den Themenkomplexen Infektion und Pneumonie, Immunologie, Asthma und COPD, Pulmonale Hypertonie, Remodeling wurden insgesamt 40 Kurzvorträge gehalten. Während der Tagung und der gemeinsamen Abendveranstaltung wurden die vorgestellten Ergebnisse lebhaft diskutiert und neue wissenschaftliche und persönliche Kontakte geknüpft. 3 Vorträge wurden prämiert und in einem gemeinsamen Symposium „Best of Herbsttagung“ auf dem diesjährigen DGP-Kongress vorgestellt. Gemeinsam mit der Sektion Zellbiologie wollen wir in Zukunft mit dieser Tagung weiter eine Plattform bieten, um auch den forschungsaktiven Kollegen aus anderen Sektionen die Möglichkeit zu bieten ihre Arbeit vorzustellen und zu diskutieren. Tagungsort 2015 wird München sein.

Um Konzepte der infektiologischen Ausbildung in der Pneumologie weiter zu fördern, wurde der Workshop „Pneumologische Infektiologie“ in Kooperation mit der Westdeutschen Gesellschaft für Pneumologie (WDGP) im November 2014 unter der Leitung von Prof. Joachim Lorenz in Lüdenscheid erneut durchgeführt.

Auf der Sektionssitzung während des Kongresses in Berlin wurden turnusgemäß die Sprecher der Sektion 4 gewählt. Neuer Sprecher ist PD Dr. Daniel Drömann, Lübeck, stellvertretender Sprecher Dr. Felix Ringshausen, Hannover. Wir danken Prof. Stefan Krüger, Düsseldorf, für die erfolgreichen letzten 4 Jahre als Sprecher bzw. stellvertretender Sprecher der Sektion.

PD Dr. Daniel Drömann, Lübeck, und Dr. Felix Ringshausen, Hannover 
Sektion 5

\section{Intensiv- und Beatmungsmedizin}

Die in den letzten Jahren bereits eingeleitete verstärkte Ausrichtung der Sektion 5 Intensiv- und Beatmungsmedizin auf die Intensivmedizin wurde weitergeführt. Das zeigte sich u.a. an dem Schwerpunkt Extrakorporale Lungenersatzverfahren zur Verbesserung von Oxygenierung und Kohlendioxid-Elimination sowohl in den Postgraduiertenkursen als auch in den Symposien des diesjährigen Kongresses in Berlin. Daneben hat das prolongierte Weaning weiterhin seinen festen Stellenwert in der Sektion 5, u. a. durch Integration der Gruppe 5.1. WeanNet (ehemals AG WeanNet) in die Sektion. Durch Neuwahl der Sprecher (Sprecher: Dr. Michael Westhoff, Hemer; Stellvertretender Sprecher Prof. Stefan Kluge, Hannover) wurde auch eine gemeinsame Führung von Sektion und Gruppe 5.1. (Sprecher Prof. Bernd Schönhofer, Stellvertretender Sprecher Dr. Jens Geiseler, Gauting), die passager durch die Neuentwicklung bedingt war, wieder aufgehoben. Aus diesen Gründen erscheint die Ausrichtung der Sektion 5 sowohl in Richtung Beatmungs- als auch in Richtung Intensivmedizin folgerichtig: neben typischen Weaning- und Beatmungsthemen werden speziell intensivmedizinische Themen auch in Zukunft einen wesentlichen Teil der Kongressaktivitäten einnehmen.

\section{Leitlinien / Empfehlungen}

Im Dezember 2014 konnte die unter Federführung der DGP seit 5 Jahren in Arbeit befindliche S2k-Leitlinie „Prolongiertes Weaning“ in Form einer Pocketversion finalisiert und publiziert werden.

Die Überarbeitung der S3-Leitlinie „Nichtinvasive Beatmung als Therapie der akuten respiratorischen Insuffizienz" ist weit vorangeschritten, die finale Fassung ist von den meisten Fachgesellschaften verabschiedet worden, lediglich die Voten der Deutschen Gesellschaft für Anästhesiologie und Intensivmedizin (DGAI) und der DGP fehlen noch. Die Freigabe zur Publikation wird im 2. Quartal 2015 erwartet.

Die Gültigkeit der S2-Leitlinie „Nichtinvasive und invasive Beatmung bei chronisch respiratorischer Insuffizienz“ ist bis Ende
2014 verlängert worden. Prof. Wolfram Windisch, Köln, wurde von der DGP mit der Revision der Leitlinie beauftragt, die aufgrund der dramatischen Veränderungen in den letzten Jahren in den außerklinischen Strukturen in ihren Inhalten angepasst und gemeinsam mit der Deutschen Interdisziplinären Gesellschaft für außerklinische Beatmung (DIGAB) und weiteren beteiligten Fachgesellschaften erarbeitet werden wird. Die Entwürfe für die erste Konsensuskonferenz werden gerade erstellt, die erste Konsensuskonferenz ist für Juni 2015 geplant.

\section{Curriculum Beatmungsmedizin $\nabla$}

In Gauting wurde im Juni 2014 erneut das Curriculum Beatmung für Bayern abgehalten, die nächste Veranstaltung in Nordrhein-Westfalen wird im April unter der Leitung von Dr. Clemens Kelbel in Dortmund durchgeführt. Aus Rückmeldungen von beiden Standorten lässt sich eine sehr positive Annahme der Veranstaltung ableiten. Der Wunsch der Sektion ist, dass weitere Kliniken lokal in anderen Bundesländern ebenfalls Curricula für Beatmungsmedizin durchführen.

\section{Herbsttagung 2014 \\ $\nabla$}

Die Herbsttagung der Gesellschaft fand dieses Jahr gemeinsam mit der Sektion Respiratorisches Versagen der Deutschen Gesellschaft für Internistische Intensivmedizin und Notfallmedizin (DGIIN) in Köln im November statt. Schwerpunktthemen waren extrakorporale Lungenersatzverfahren, Lebensqualität auf der Intensivstation, Pathophysiologie von Atmung und Beatmung und hyperkapnisches Lungenversagen. Die Besucherzahl der Veranstaltung war ausgezeichnet. Am 13. und 14. November 2015 wird die Herbsttagung 2015 gemeinsam mit der Sektion 15 Atmungstherapeuten und Gesundheitsberufe in Greifswald stattfinden.

\section{ARDS-Netzwerk} $\nabla$

Mehrere Mitglieder der Sektion sind im deutschen ARDS-Netzwerk aktiv. Seit wenigen Monaten wird in diesem Rahmen die multizentrische deutsche DACAPO-Studie durchgeführt, um die Versorgungsqualität und individuelle Patientenmerkmale auf die gesundheitsbezogene Lebensqualität bei ARDS-Überlebenden zu untersuchen.

\section{Kooperationen \\ $\nabla$}

Kooperationen mit der DIVI (Sprecher der Sektion Respiratorisches Versagen: Prof. Bernd Schönhofer) und der DGIIN (Präsident: Prof. Stefan Kluge) bestehen weiterhin, in Form von Mitgestaltung der jeweiligen Kongressprogramme sowie Beteiligung an der Erstellung von Positionspapieren/ Leitlinien.

Im Bereich außerklinische Beatmung besteht eine intensive Zusammenarbeit mit der DIGAB, u.a. sichtbar in der Organisation des Beatmungssymposiums im Rahmen der Jahrestagungen der DIGAB.

Im November 2014 fand in Hannover erneut der Kurs der ERS-School „Non-invasive Ventilation: Basic Concepts“ statt.

Auf internationaler Ebene sind 3 Mitglieder der Sektion weiterhin am HERMESProjekt „Respiratory Critical Care“ der ERS beteiligt. Nach Finalisierung von Syllabus und Curriculum wird gerade der Multiple-Choice-Fragenkatalog für die für September 2016 geplante ersten schriftliche Examen erstellt.

\section{Gruppe 5.1. WeanNet}

$\nabla$

In der Gruppe 5.1. wurde eine erste Überblicks-Publikation zu den zertifizierten Weaning-Zentren in der Zeitschrift Pneumologie im Jahr 2014 veröffentlicht. Eine englischsprachige erste Auswertung der Datenbank (aktuell sind ca. 10000 Patienten im prolongierten Weaning erfasst) zu Epidemiologie und Outcome des prolongierten Weanings ist gerade in der Finalisierungsphase. Autor wird die WeanNet Study Group sein. Die Zahl der in die Datenbank eingebenden Zentren hat leicht zugenommen. Mittlerweile gibt in jedem Bundesland mindestens ein Zentrum seine Daten in die vom Institut für Lungenforschung verwaltete Datenbank ein.

Gegen die zunehmende Bewerbung des außerklinischen Weanings in Pflegeheimen ist die kurzfristige Erstellung eines Positionspapier gemeinsam mit weiteren Gesellschaften wie DIGAB, Verband pneumologischer Kliniken (VPK), DGIIN, Bundesverband der Pneumologen (BdP) und auch mit Vertretern der Kostenträger wie der MDS geplant.

Dr. Michael Westhoff, Hemer, und Prof. Stefan Kluge, Hamburg 


\section{Sektion 6}

\section{Kardiorespiratorische Interaktion}

Die Sektion Kardiorespiratorische Interaktionen bietet für diejenigen ein klinisches und wissenschaftliches Forum, die sich mit Störungen des Zusammenwirkens von Lunge und Herz beschäftigen oder auch an den pathophysiologischen Grundlagen interessiert sind. In den letzten Jahren gab es hier sowohl bei der akuten Lungenembolie als auch bei der chronischen pulmonalen Hypertonie und bei anderen Lungenerkrankungen mit Auswirkungen auf die kardiale Funktion entscheidende Erkenntnisse in Bezug auf Diagnostik und Therapie.

Mitglieder der Sektion Kardiorespiratorische Interaktion haben sich, wie auch in den Vorjahren, aktiv in die Gestaltung des Kongresses 2015 in Berlin und die Planung des Kongresses 2016 eingebracht. Organisiert wurden von der Sektion 3 Postgraduiertenkurse, 2 Frühseminare und 6 Symposien, 4 davon in enger Kooperation mit anderen Sektionen. An weiteren Symposien und PG-Kursen war die Sektion 6 als Kooperationspartner beteiligt. Themen waren u.a. Echokardiografie, Spiroergometrie, akute Lungenembolie, Dyspnoe, pulmonale Hypertonie und kardiorespiratorische Funktionsdiagnostik. Zum 3. Mal wurde ein aufwendiger „Hands on“ Rechtsherzkatheter-Kurs durchgeführt.

Bei dem Herbsttreffen der Sektion im November 2014 in der Universitätsmedizin Göttingen erfolgte die Diskussion aktueller Projekte von Mitgliedern, die Planung des Jahreskongresses sowie Neuwahlen der Sektionssprecher. Neben den Übersichtsvorträgen zu aktuellen Fragen konnten insbesondere auch jüngere Mitglieder der Sektion in Kurzvorträgen wissenschaftliche Ergebnisse ihrer Arbeit vortragen. Aus 9 qualitativ hochwertigen, eingereichten und vorgetragenen wissenschaftlichen Projekten wurde auf dem Herbsttreffen 2014 die beste ausgewählt und erstmals mit einem Posterpreis der Sektion über $1000 €$ (von Almirall eingeworben) ausgezeichnet. 2015 wird das Herbsttreffen der Sektion 6 in Homburg a.d. Saar stattfinden.

In der „Pneumologie“ wurden 2014 insgesamt 10 Artikel publiziert, an denen Mitglieder der Sektion 6 beteiligt waren. Zusammen mit der AG Tabakprävention wur- de die S3-Leitlinie „Tabakentwöhnung bei COPD“ (Pneumologie 2014; 68: 237-258) erstellt. Daneben handelt es sich um ein Konsensuspapier, ein Positionspapier, 3 Originalarbeiten sowie 4 Übersichten.

Mitglieder der Sektion 6 waren in der Arbeitsgemeinschaft „PH-DACH“ (von Experten aus Deutschland, Österreich und der Schweiz) der Deutschen Gesellschaft für Kardiologie und der Deutschen Gesellschaft für Pneumologie und Pädiatrischen Kardiologie an der Erstellung von 2 Positionspapieren und 7 Manuskripten zu verschiedenen Aspekten von Diagnostik und Therapie der Pulmonalen Hypertonie beteiligt, die 2014 in einem DMW-Sonderheft publiziert wurden.

Bei der Vielzahl von Aufgaben sind neue Mitglieder jederzeit willkommen, jüngere Mitglieder werden gezielt gefördert.

Prof. Heinrike Wilkens, Homburg, und PD Dr. Mathias Borst, Bad Mergentheim

\section{Sektion 7}

\section{Klinische Pneumologie}

Die Sektion Klinische Pneumologie ist eine zentrale Schnittstelle zwischen klinischer Forschung, Grundlagenforschung und translationaler Anwendung neuer Forschungsergebnisse in der klinischen Praxis. Nicht zuletzt aus diesem Grunde ist sie die mitgliederstärkste und für viele die interessanteste wissenschaftliche Sektionen der DGP. Die zunehmende Emanzipation der klinischen Forschung als eigenständige Forschungsrichtung verstärkt den wissenschaftlichen Charakter der Sektion. Ungeachtet dessen ist die Sektion klinische

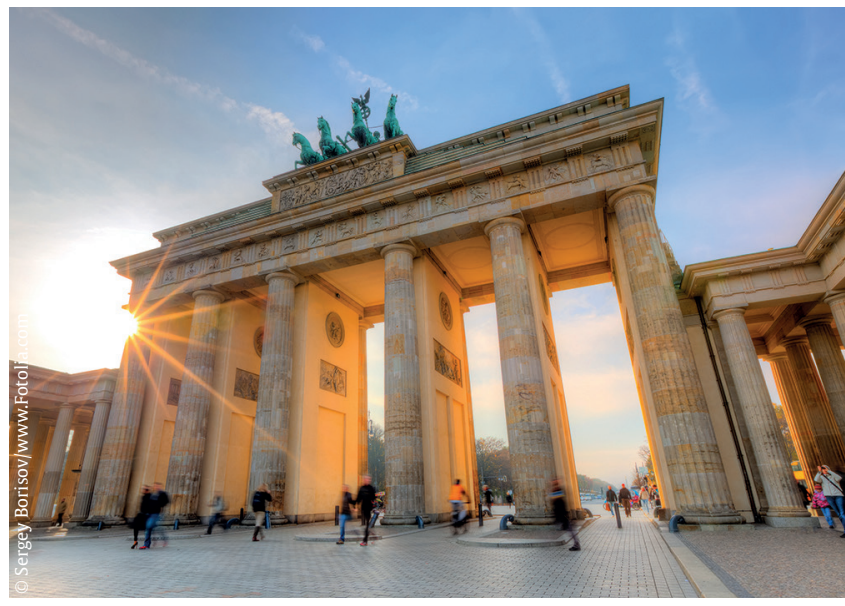

Pneumologie aber auch die Plattform für den Austausch über Einzelfälle und seltene Krankheitsbilder, die nicht selten auch den Anstoß für neue wissenschaftliche Überlegungen und klinische Studien geben. Die Sektion Klinische Pneumologie repräsentiert daher in besonderer Weise die gesamte Breite der Pneumologie und bietet Anknüpfungspunkte für praktisch alle anderen Sektionen der DGP, was sich auch in einer sehr starken Vernetzung und den Kooperationen widerspiegelt.

Der Hauptstadtkongress in Berlin war auch für die Sektion Klinische Pneumologie ein großer Erfolg. Mit sehr gut besuchten Symposien und einem insgesamt positiven Feedback durch die Kongressteilnehmer. Die Lebendigkeit der Sektion spiegelt sich auch in der seit Jahren steigenden Anzahl von eingereichten Abstracts wider. So waren es für Berlin über 100 Abstracts, von denen 97 akzeptiert und die in Vorträgen oder Postersitzungen präsentiert wurden, wodurch auch dem klinischen Nachwuchs ein interaktives Forum geboten werden konnte. Insgesamt 31 Symposien wurden von der Sektion 7 gestaltet, davon 28 in Kooperation mit einer oder mit mehreren anderen Sektionen und Arbeitsgruppen. Hinzu kommen 9 Postgraduiertenkurse (davon 7 in Kooperation) und ein Frühseminar. Im Vergleich zu Bremen im Jahr 2014 stieg die Zahl der Sitzungen mit freien Vorträgen von 3 auf 6 und die Zahl der Postersitzungen von 5 auf 7. Die Besucherzahlen der Veranstaltungen können als durchweg hervorragend bezeichnet werden, teilweise waren es über 250 Teilnehmer.

Als weitere Aktivitäten mit zentraler Beteiligung der Sektion Klinische Pneumologie sind das Bad Reichenhaller Kolloquium und der Kongress der Wissenschaftlichen Arbeitsgruppe für die Therapie von

Rund 4000 Besucher konnte der Kongresspräsident Prof. Torsten Bauer in der deutschen Hauptstadt begrüßen. Das diesjährige Motto lautete „Gemeinsam für den Patienten“. 
Lungenerkrankungen (WATL) zu nennen. Das Bad Reichenhaller Kolloquium ist eine Traditionsveranstaltung, das am 19. und 20. Juni 2015 die „akute Exazerbation“ von Lungenerkrankungen zum Thema hat und dies aus unterschiedlichsten Blickwinkeln beleuchten wird. Es werden hier über 600 Teilnehmer erwartet. Der WATL-Kongress wird in 2-jährigen Intervallen durchgeführt und am 22. und 23. Januar 2016 in Berlin unter dem Motto „Von seltenen Lungenerkrankungen lernen“ stattfinden. Traditionell ist der WATL-Kongress den seltenen und sehr seltenen Lungenerkrankungen gewidmet, die sonst oft in der Konkurrenz $\mathrm{zu}$ den pneumologischen Volkskrankheiten zu wenig Beachtung finden. Andererseits sind es oft die seltenen Lungenkrankheiten, die ein Schlaglicht auf die Pathogenese und Pathophysiologie von Lungenerkrankungen werfen und so neue Perspektiven eröffnen.

Für den kommenden DGP-Kongress in Leipzig 2016 laufen die Vorbereitungen innerhalb der Sektion bereits auf Hochtouren. Im Rahmen der Sektionssitzung in Berlin wurden eine Vielzahl interessanter Symposiumsvorschläge eingebracht und diskutiert, sodass wieder mit einer äußerst aktiven Beteiligung der Sektion 7 gerechnet werden darf.

Mitglieder der Sektion Klinische Pneumologie sind wesentlich an der Durchführung und Auswertung der Daten des COSYCO-Nets beteiligt, dem Kompetenznetz für obstruktive Atemwegserkrankungen, Asthma und COPD des BMBF. Hier werden regelmäßig Investigator Meetings ausgerichtet. Auch der Aufbau von klinischen Registern für seltene Erkrankungen, wie z. B. Lungenfibrose, Lymphangioleiomyomatose und Langerhanszell Histiozytose wird maßgeblich von Mitgliedern der Sektion vorangetrieben.

In der Fachzeitschrift „Pneumologie“ der Gesellschaft wurden 2014 insgesamt 11 Artikel von Mitgliedern der Sektion 7 oder unter deren Beteiligung publiziert. Neben 3 Konsensuspapieren waren es 4 Originalund 4 Übersichtsarbeiten.

Insgesamt war das Jahr 2014 für die Sektion klinische Pneumologie sehr erfolgreich mit einer weiteren Steigerung gegenüber 2013.

Prof. Jürgen Behr, München, und Prof. Jens Schreiber, Magdeburg
Sektion 8

\section{Schlafmedizin}

Das Sektionstreffen der Sektion 8 fand wie schon in der Vergangenheit gemeinsam mit der Sektion Apnoe der Deutschen Gesellschaft für Schlafforschung (DGSM) und der Sektion 35 der DGK mit mehr als 50 wissenschaftliche Teilnehmern und 22 Vorträgen in Hagen statt. Besonders hinzuweisen ist auf die konstruktive Zusammenarbeit mit der Deutsche Gesellschaft für Zahnärztliche Schlafmedizin (DGZS). Auf dem Treffen wurde deutlich, dass zahlreiche wissenschaftliche Projekte im Bereich der Schlafmedizin durchgeführt werden.

Auf dem diesjährigen DGP-Kongress in Berlin war die Sektion Schlafmedizin mit 6 Symposien und einem Postgraduiertenkurs gut vertreten. Die Rückmeldung aus den Symposien war sehr positiv, auch die wissenschaftlichen Vorträge waren gut besucht. Für den DGP-Kongress 2016 in Leipzig wird die Sektion 2 Postgraduiertenkurse und 6 Symposien anbieten. Als Themen sind geplant: „Pathophysiologie der Schlafapnoe“, „Pathophysiologie der Hyperkapnie“, „Verkehrsmedizin/Umsetzung der EU-Richtlinie“, ,Schlafstörungen bei Frauen“, „Chronisch-ventilatorische Insuffizienz und Schlaf“, „Der gefährdete Schlafapnoe-Patient“, „Einführung in die Schlafmedizin“ und „Beatmung im Schlaf bzw. nächtliche Beatmung“.

Auf dem Sektionstreffen in Berlin wurde auch über die zukünftige inhaltliche Ausrichtung diskutiert: In den meisten pneumologischen Kliniken werden zunehmend Patienten mit chronischem hyperkapnischen Atemversagen auf NIV eingestellt. Damit stellt die chronische Atmungsinsuffizienz einen wissenschaftlichen Schwerpunkt der Sektion 8 dar. Diese Schwerpunktsetzung sollte auch in den Kongressen deutlicher werden. Möglicherweise ist hierfür auch eine Namensänderung der Sektion notwendig. Damit keine Unstimmigkeiten zwischen den Sektionen aufkommen, sollten Gespräche mit der Sektion Intensiv- und Beatmungsmedizin geführt werden. Joint-Meetings und -Symposien sollten ebenfalls angestrebt werden.

Das nächste Sektionstreffen (früher SNAKTagung) soll am 29. und 30. Januar 2016 in Regensburg bei Prof. Michael Arzt stattfinden. Die Sektion plant weiterhin ein „Brainstorming-Treffen“ zur Vorbereitung eines gemeinsamen Forschungsantrags für das Jahr 2015 an einem „ruhigen Platz“ (Organisation Prof. Nikolaus Netzer). Falls sich kein Termin finden lassen sollte, wäre auch ein Treffen im Anschluss an die SNAKTagung in Regensburg zu überlegen.

Die Schlafmedizin steht in einem besonderen gesundheitspolitischen Spannungsfeld. Alle wissenschaftlichen Untersuchungen zur Epidemiologie schlafbezogener Atmungsstörungen zeigen eine hohe Bedeutung der Erkrankungen mit einer Zunahme der Prävalenz infolge der Adipositas-Epidemie. Eine effektive SBAS-Therapie reduziert die Folgekosten für Sekundärerkrankungen. Demgegenüber stehen die Bemühungen der Kostenträger, die durch die Therapie und Diagnostik ansteigenden Ausgaben $\mathrm{zu}$ begrenzen. Aktuell wird durch die Kostensenkungen die ambulante und stationäre Diagnostik und Therapie der Patienten bedroht und auch die weitere Versorgung, da infolge der Ausschreibungen der Hilfsmittel, eine qualitativ einwandfreie Versorgung bedroht ist. Eine gute Akzeptanz der Überdrucktherapie kann nur durch eine hochwertige Patientenbetreuung erreicht werden. Die Mitglieder der Sektion machen sich daher große Sorge, dass sich die Qualität der Patientenversorgung in den nächsten Jahren verschlechtern wird. Die Sektion 8 hat sich daher sowohl an der Erstellung eines Positions- als auch eines Konsenuspapiers zur Situation der Schlafmedizin beteiligt. Weiterhin wurde ein Positionspapier zu rechtlichen Fragen bezüglich der Versorgung von Schlafapnoe-Patienten mit CPAP oder anderen Überdrucktherapien bei einem Krankenhausaufenthalt erstellt.

Unter Berücksichtigung der o.g. besonderen Situation fand am 20. Februar 2015 ein Treffen von BdP, DGSM, VPK und DGP zum Thema „Schlafmedizin“ in Frankfurt a.M. statt. Auf dem Treffen wurden Ideen für zukünftige Strukturen und zur weiteren Finanzierung der Schlafmedizin in Deutschland, mit dem klaren Schwerpunkt der obstruktiven Schlafapnoe, besprochen.

Weiterhin erwähnenswert ist, dass Prof. Winfried Randerath, Solingen, zum Leiter der ERS-Gruppe „Sleep Medicine“ und Prof. Jürgen Fischer, Norderney, zum Ehrenmitglied der Tschechischen Gesellschaft für Schlafmedizin gewählt wurden.

PD Dr. Georg Nilius, Hagen, und Prof. Kurt Rasche, Wuppertal 